\title{
Pregnancy outcomes in pulmonary arterial hypertension in the modern management
}

\section{era}

\author{
Xavier Jaïs*,\#,ף,+, Karen M. Olsson ${ }^{+,++}$, Joan A. Barbera ${ }^{\S}$, Isabel Blanco ${ }^{\S}$, \\ Adam Torbicki ${ }^{f}$, Andrew Peacock**, C. Dario Vizza ${ }^{\# \#}$, Peter Macdonald ${ }^{\text {}}$, \\ Marc Humbert ${ }^{\star, \#, \oplus,++}$ and Marius M. Hoeper ${ }^{+,++}$
}

ABSTRACT: Previous studies have reported mortality rates of up to $56 \%$ associated with pregnancy in pulmonary arterial hypertension (PAH) but the management of this disease has changed considerably in recent years.

We compiled a multinational, prospective registry to examine the contemporary outcome of pregnancies in patients with $\mathrm{PAH}$.

During a 3-yr period, the 13 participating centres reported 26 pregnancies. Three (12\%) females died and one (4\%) developed right heart failure requiring urgent heart-lung transplantation. There were eight abortions; two spontaneous and six induced. $16(62 \%)$ pregnancies were successful, i.e. the females delivered healthy babies without complications. These females had well controlled PAH (pulmonary vascular resistance (PVR) $500 \pm 352 \mathrm{dyn} \cdot \mathrm{s} \cdot \mathrm{cm}^{-5}$ ); eight of them were long-term responders to calcium channel blockers. In contrast, the females who died or required transplantation had poorly controlled PAH (PVR 1,667 $\pm 209 \mathrm{dyn} \cdot \mathrm{s} \cdot \mathrm{cm}^{-5}$ ).

Pregnancy remains associated with a substantial mortality rate in PAH. However, our results indicate that the outcome of pregnancy in PAH has improved, at least when PAH is well controlled, and particularly in long-term responders to calcium channel blockers. These data must be confirmed by larger series before the general recommendation to avoid pregnancy in all patients with $\mathrm{PAH}$ is reconsidered.

KEYWORDS: Hypertension, pregnancy, pulmonary, right heart failure

$\mathbf{T}$ he term pulmonary arterial hypertension (PAH) describes a group of diseases characterised by progressive obstructive pulmonary vasculopathy almost invariably resulting in rapid right heart failure and death if not sufficiently treated. Although PAH can occur in males and females of all age groups, the disease is frequently encountered in younger females [1, 2]. Female patients with $\mathrm{PAH}$ may become pregnant and occasionally the first clinical manifestations of $\mathrm{PAH}$ are seen during the course of pregnancy. It is well known that pregnancy poses an immense risk to females with PAH. In a systematic analysis of reports published between 1978 and 1996, WEISS et al. [3] calculated maternal mortality rates of $36 \%$ in Eisenmenger's syndrome, $30 \%$ in primary pulmonary hypertension (now called idiopathic pulmonary arterial hypertension (IPAH)) and 56\% in pulmonary hypertension associated with other conditions. Neonatal mortality was also substantial, ranging from $11 \%$ to $13 \%$ [3]. Therefore, current guidelines unanimously recommend that pregnancy be avoided or terminated early in females with PAH $[4,5]$.

The treatment of PAH has undergone considerable changes in recent years. Several classes of substances including calcium channel blockers, prostacyclin derivatives, endothelin receptor antagonists and phosphodiesterase- 5 inhibitors are now being widely used in a tailored approach resulting in improved haemodynamics, exercise capacity and outcome [6-10]. Although it is generally not possible to cure $\mathrm{PAH}$ with these medications, some patients achieve substantial improvement and long-term stabilisation with near-normal haemodynamics. This is particularly true for so-called "responders", i.e. patients with marked vasoreactivity who can be treated successfully with high-dose calcium channel blockers [11].

There is emerging evidence from recent case series that the outcome of pregnancy in females with $\mathrm{PAH}$ may be better in the modern management era [12-14]. A recent systematic review examined
AFFILIATIONS

*Univ. Paris-Sud, Faculté de Médecine, Le Kremlin-Bicêtre.

${ }^{\#}$ Hôpital Antoine Béclère, Assistance

Publique Hôpitaux de Paris.

"INSERM U999, Hôpital Antoine

Béclère, Clamart, France.

+Hannover Medical School,

Hannover, Germany.

${ }^{\S}$ Hospital Clinic, CIBERES, University

of Barcelona, Spain.

fPostgraduate Medical School,

Warsaw, Poland.

**University of Glasgow, Glasgow,

UK.

\#\# Sapienza University of Rome,

Rome, Italy.

"'St Vincent's Hospital, Sydney,

Australia.

${ }^{++}$These authors contributed equally.

CORRESPONDENCE

M.M. Hoeper

Hannover Medical School

Dept of Respiratory Medicine

30623 Hannover

Germany

E-mail: hoeper.marius@mh-

hannover.de

Received:

Aug 172011

Accepted after revision:

Dec 262011

First published online:

Jan 262012 
the reported outcomes of pregnancy in patients with PAH in 1997-2007. Overall, maternal mortality was lower in that period compared with previous eras $(p=0.047)$. Mortality decreased substantially in all three subgroups investigated (from 30\% to $17 \%$ in IPAH, from $36 \%$ to $28 \%$ in Eisenmenger's syndrome and from $56 \%$ to $33 \%$ in PAH of other aetiologies) [13].

In order to obtain further data on the outcome of pregnancy in females with $\mathrm{PAH}$ in the modern management era, we launched an international, non-interventional registry that prospectively collected data over a 3-yr period.

\section{METHODS}

The present survey was designed to prospectively collect data from pregnancies occurring between July 1, 2007 and June 30, 2010 in females with PAH. 13 pulmonary hypertension centres in Europe, USA and Australia agreed to participate. Inclusion criteria were age $\geqslant 18$ yrs and a diagnosis of $\mathrm{PAH}$ according to established diagnostic criteria including right heart catheterisation [4]. Patients with other forms of pulmonary hypertension were excluded. The participating centres received a structured questionnaire to be filled out for each case of pregnancy, regardless of the outcome. Data were collected regarding the type of PAH, medical therapy and disease severity at the time when pregnancy was discovered (including the most recent haemodynamic data), follow-up and medical therapy during pregnancy, time and management of delivery, peripartum and postpartum management, and maternal and neonatal outcome. Patients who had a successful pregnancy were followed for 12 months.

The protocol was approved by the institutional review boards of the participating centres. Patient data were made anonymous. Written informed consent was obtained from patients if possible but was waived when the patient's medical condition made informed consent impossible.

\section{Statistical analysis}

Data are presented as mean \pm SD for parametric data. All analyses were descriptive as the small number of observations did not allow in-depth statistical analyses.

\section{RESULTS}

Of the 13 participating centres, six did not observe pregnancies among their PAH patients during the study period. The remaining seven centres contributed data on 26 pregnancies. The baseline characteristics of the females who became pregnant during the observation period are shown in table 1 . The followup periods after delivery or pregnancy termination ranged from 1 to 36 months (median, 23 months).

\section{Maternal and neonatal outcomes}

$18(69 \%)$ females delivered healthy babies (mean time \pm SD of delivery, $33.9 \pm 3$ weeks). One of these females, however, developed right heart failure and required extracorporeal life support followed by urgent heart-lung transplantation. Another patient delivered a healthy baby but died shortly thereafter from refractory right heart failure. For the purpose of the following analyses, $16(62 \%)$ pregnancies were considered successful in that mother and baby survived without complications. There were eight abortions; two occurred spontaneously at gestational week 9 and 12, respectively; the other six were induced (mean

TABLE 1 $\begin{aligned} & \text { Baseline characteristics of patients with } \\ & \text { pulmonary arterial hypertension (PAH) }\end{aligned}$
pregnancies

Data are presented as $n$, mean \pm SD or $n(\%)$. NYHA FC: New York Heart Association functional class; 6MWD: 6-min walk distance; Pra: right arterial pressure; mPAP: mean pulmonary artery pressure; $P$ pcw: pulmonary capillary wedge pressure; $\mathrm{Cl}$ : cardiac index; PVR: pulmonary vascular resistance; $\mathrm{Sv}, \mathrm{O}_{2}$ : mixed venous oxygen saturation; PDE: phosphodiesterase. ${ }^{\#}$ : at the time pregnancy was discovered; " : most recent variables at the time pregnancy was discovered; ${ }^{+}:$in these patients the diagnosis of PAH was made during pregnancy.

gestational weeks $\pm S D, 10 \pm 3$ ). All six females with induced abortions survived without medically significant complications whereas both females with spontaneous abortions died. Thus, a total of three $(12 \%)$ females died during the early postpartum period, all of them from right heart failure. All of these females suffered from severe pulmonary hypertension (table 2) and all of them had undergone general anaesthesia for delivery or curettage, respectively.

Table 2 depicts the baseline characteristics of females who had successful pregnancies $(n=16 ; 62 \%)$ and those who died $(n=3$; $12 \%)$ or required urgent heart-lung transplantation $(n=1 ; 4 \%)$, respectively. Although the small number of patients in the latter group precluded statistical comparisons, the females who died or required transplantation evidently had more 
TABLE 2 Baseline characteristics (at the time when pregnancy was discovered) of females who delivered a healthy baby and survived, of females with induced abortions, and of females who did not survive pregnancy or required transplantation

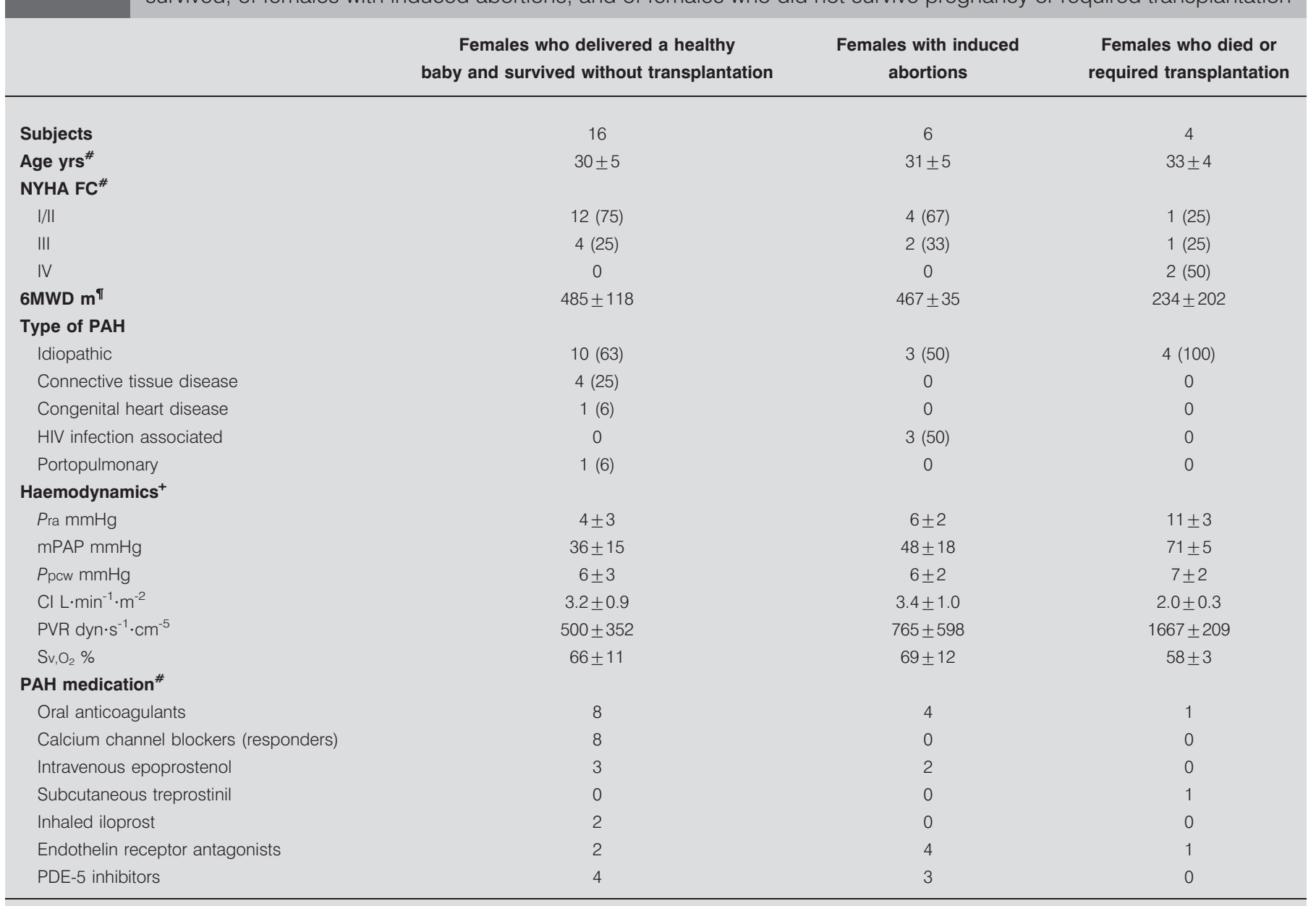

Data are presented as n, mean \pm SD or $n(\%)$. NYHA FC: New York Heart Association functional class; 6MWD: 6-min walk distance; PAH: pulmonary arterial hypertension; Pra: right arterial pressure; mPAP: mean pulmonary artery pressure; Ppcw: pulmonary capillary wedge pressure; Cl: cardiac index; PVR: pulmonary vascular resistance; $\mathrm{Sv}, \mathrm{O}_{2}$ : mixed venous oxygen saturation; PDE: phosphodiesterase. ${ }^{*}$ : at the time pregnancy was discovered; ${ }^{\circledR}$ : $6 \mathrm{MWD}$ was set to 0 m in NYHA FC IV patients unable to walk $(n=2) ;^{+}$: most recent variables at the time pregnancy was discovered

severe pulmonary hypertension than those with successful pregnancies and delivered by emergency caesarean section under general anaesthesia.

Indeed, eight (50\%) out of the 16 females who had successful pregnancies were so-called "responders" and had nearly normal haemodynamic parameters with calcium channel blocker therapy (mean pulmonary artery pressure (mPAP) $30 \pm 6 \mathrm{mmHg}$, pulmonary vascular resistance (PVR) $387 \pm 181 \mathrm{dyn} \cdot \mathrm{s}^{-1} \cdot \mathrm{cm}^{-5}$ ). The other females with successful pregnancies were nonresponders but most of them also had well-controlled PAH while receiving targeted therapy (table 2).

In contrast, the four females who died or required transplantation had poorly controlled PAH with PVR $1,667 \pm 209 \mathrm{dyn} \cdot \mathrm{s}^{-1} \cdot \mathrm{cm}^{-5}$ and $\mathrm{mPAP} 71 \pm 5 \mathrm{mmHg}$ (table 2).

The baseline characteristics of the six females with induced abortions are also shown in table 2. Most of these patients had reasonably well controlled $\mathrm{PAH}$ although there was a trend towards more severe disease than in those with successful pregnancies. In all of these cases, the physician advised terminating the pregnancy. Abortion was medically induced in all cases and surgical interventions were not required. None of these females experienced complications or worsening of pulmonary hypertension during follow-up.

Maternal outcome during the year following delivery was analysed for the 16 females who had successful pregnancies. Two (13\%) of them experienced clinical deterioration requiring intensification of PAH therapy. Neither was a long-term responder to calcium channel blockers.

\section{Management of successful pregnancies}

PAH medication during pregnancy

In the females with successful pregnancies, background treatment with calcium channel blockers, prostanoids and sildenafil was continued in all cases (table 2). Endothelin receptor antagonists were withdrawn when pregnancy was discovered. In 
two patients, inhaled iloprost was introduced as additional PAH therapy during pregnancy.

\section{Anticoagulation during pregnancy}

Five patients never received anticoagulation prior to or during pregnancy. Eight patients had their oral anticoagulants replaced by heparin during pregnancy. Three patients had not been treated with anticoagulants before they became pregnant but received heparin during their pregnancy. No thromboembolic events were observed in any of these patients.

\section{Assessments during pregnancy}

During pregnancy, the patients were regularly seen at their pulmonary hypertension centres, usually at 2-4-weekly intervals. Echocardiography was utilised as follow-up tool in all patients; 6-min walk tests and biomarkers (brain natriuretic peptide) were used in eight (50\%) and seven (43.8\%) out of 16 patients, respectively. Only one centre (French reference centre for pulmonary hypertension) performed right heart catheterisations (up to three procedures in one patient) as part of their regular assessment during pregnancy.

\section{Management of delivery and postpartum period}

One female had a preterm spontaneous vaginal delivery at week 26; mother and child had an uncomplicated clinical course. The other successful pregnancies (15 out of $16 ; 94 \%$ ) were delivered by planned caesarean section between the 31th and 38th gestational weeks; 12 (80\%) out of 15 under spinal anaesthesia and three $(20 \%)$ out of 15 under general anaesthesia. Maternal monitoring during delivery was generally non-invasive except for two occasions when haemodynamics were monitored by right heart catheterisation during delivery. There were no serious complications during delivery and no patient required prolonged mechanical ventilation or catecholamines.

\section{DISCUSSION}

This survey has two major results. First, pregnancy remains associated with a high risk in females with PAH. In this series of 26 patients, three $(12 \%)$ died and another one $(4 \%)$ had refractory right heart failure and could be rescued only with extracorporeal cardiopulmonary support followed by urgent heart-lung transplantation. Second, our data suggest that pregnancies can be successfully managed in highly selected patients with well controlled PAH. This seems to be particularly true for so-called "responders", i.e. patients with a vasoreactive form of pulmonary hypertension in whom haemodynamics become normal or almost normal with calcium channel blocker therapy [11]. This survey followed eight such "responders" and all of them had uncomplicated courses of pregnancy. The same was true for eight further patients who did not fulfil the responder criteria but had well controlled disease with PAHtargeted therapy. These results stand in contrast to previously reported mortality rates of between $30 \%$ and $56 \%$, derived between 1978 and 1996 [3], i.e. at a time when PAH-targeted therapies were not readily available [15-17]. The seemingly better outcomes of pregnancies documented in our survey might be attributed at least partly to better disease control with current remedies. Our results are comparable with those observed by BÉDARD et al. [13] who reported mortality rates between $17 \%$ and $33 \%$ in $1997-2007$.
In our series, the four females who died or required transplantation had very severe PAH, with PVR ranging from $1,560 \mathrm{dyn} \cdot \mathrm{s}^{-1} \cdot \mathrm{cm}^{-5}$ to $1,928 \mathrm{dyn} \cdot \mathrm{s}^{-1} \cdot \mathrm{cm}^{-5}$, respectively. These findings are consistent with those reported by ROBERTS and KEAST [18], who were able to provide some prognostic data on pregnant patients with PAH using haemodynamics values, and demonstrated that patients with a PVR $>1,000 \mathrm{dyn} \cdot \mathrm{s}^{-1} \cdot \mathrm{cm}^{-5}$ had the worst prognosis.

In addition, the females having a worse outcome in our study delivered by emergency caesarean section under general anaesthesia, and worsened and/or died during the early postpartum period. BÉDARD et al. [13] made the same observations, and showed that general anaesthesia was associated with a four-fold increase in maternal mortality and that the majority of deaths occurred mainly within the first month after delivery. The high mortality rate recorded in patients who underwent general anaesthesia might be explained by a downwards spiral of worsening right ventricular function. Indeed, general anaesthesia may increase PVR through several mechanisms including increased sympathetic stimulation during airway instrumentation and tracheal intubation [19], effects of volatile agents used for anaesthesia [20], high airway plateau pressure due to the effects of positive-pressure mechanical ventilation [21] and hypoxic pulmonary vasoconstriction caused by alveolar hypoxia [22].

Our 3-yr prospective survey was conducted in 13 expert centres for pulmonary hypertension. Pregnancies were seen only in six of these centres, which probably reflects the fact that physicians strongly advise their PAH patients not to become pregnant. At the same time it appears that some centres have become more liberal in highly selected patients. Our data indicate that physicians in all participating centres made strong recommendations against becoming pregnant in females with severe or moderately severe pulmonary hypertension. They also recommended abortion of pregnancies whenever they felt that pulmonary hypertension was not well controlled. However, in some females with minor haemodynamic impairment and longterm stabilisation under therapy, it was decided to continue pregnancy. Notably, pregnancies were successful in all of these cases, suggesting that experienced physicians may have a good judgment on when it may be safe for females with PAH to continue pregnancy, even in the absence of supporting data.

Our study has obvious limitations, the most important one being the small sample size. However, 13 large pulmonary hypertension centres participated in this prospective 3-yr survey and we are confident that all cases were reported. Thus, the small sample size merely reflects the simple fact that pregnancies occur rarely among females with PAH. This hypothesis is supported by the results of a large US health survey reporting only 182 patients with IPAH out of 11.2 million deliveries [23].

Because of the non-interventional design and the small sample size, the present survey does not provide further guidance towards an optimised management of pregnancies in females with pulmonary hypertension. All centres continued established $\mathrm{PAH}$ therapy except for endothelin receptor antagonists as these drugs are contraindicated during pregnancy because of their teratogenic potential [24]. Anticoagulation was managed differently in all centres without an apparent effect on outcome. Planned delivery by caesarean section between gestational weeks 
35 and 38 was the preferred method in most centres but there is insufficient information to determine whether this approach provides the best outcomes for mothers and their babies.

Currently, there is an unequivocal statement in both the US and the European guidelines that pregnancy be avoided or early terminated in all females with $\mathrm{PAH}[4,5]$. Without any doubt, this recommendation remains undisputed in females with poorly controlled PAH. In selected females with wellcontrolled PAH and normal or near-normal haemodynamics, however, such decisions should be made on an individual basis after the potential risks have been openly discussed with the patient and her relatives. As there are presently insufficient data to support any cut-off values to determine when it might be safe for females with PAH to continue pregnancy and when not, such vital decisions should be made only in highly experienced referral centres. It would certainly be misleading to conclude from our data that pregnancy is now safe for females with PAH. Misinterpreting the present data thus bears the inherent danger that physicians become too liberal in females who would be better advised avoiding or terminating pregnancy. When advising patients with PAH who want to become pregnant, one should also bear in mind the impact of raising a child for females with a disease usually characterised by exercise limitation, fatigue and a reduced life expectancy.

In conclusion, our data show that the risks associated with pregnancy in patients with PAH remain substantial. In line with current guidelines, pregnancy should be avoided or early terminated in females with severe PAH. However, our data also indicate that the risks associated with becoming pregnant in females with well controlled disease and minor haemodynamic impairment may have declined. If such females become pregnant or want to become so, a thorough risk assessment in an experienced centre is necessary and the females need to be fully counselled about the potential risks before a final decision is being made.

\section{STATEMENT OF INTEREST}

Statements of interest for X. Jaïs, K.M. Olsson, A. Torbicki, A. Peacock, C.D. Vizza, P. Macdonald, M. Humbert and M.M. Hoeper can be found at www.erj.ersjournals.com/site/misc/statements.xhtml

\section{ACKNOWLEDGEMENTS}

The authors wish to thank the following persons for contributing to this survey: M. Gomberg-Maitland and D. Edwards (University of Chicago, Chicago, IL, USA); V. McLaughlin (University of Michigan, Ann Arbor, MI, USA); D. Badesch, D. McCollister and T. Bull (University of Colorado, Denver, CO, USA); I. Preston (Boston University, Boston, MA, USA); M. Halank (University of Dresden, Dresden, Germany); H-J. Seyfarth (University of Leipzig, Leipzig, Germany); G. Simonneau, O. Sitbon and F. Mercier (Paris-Sud University, Clamart, France); G. Habib and S. Renard (University of Marseille, Marseille, France); E. Hachulla (University of Lille, Lille, France); I. Frachon and C. Gut-Gobert (University of Brest, Brest, France).

\section{REFERENCES}

1 Humbert M, Sitbon O, Chaouat A, et al. Pulmonary arterial hypertension in France: results from a national registry. Am J Respir Crit Care Med 2006; 173: 1023-1030.

2 Rich S, Dantzker DR, Ayres SM, et al. Primary pulmonary hypertension. A national prospective study. Ann Intern Med 1987; 107: 216-223.
3 Weiss BM, Zemp L, Seifert B, et al. Outcome of pulmonary vascular disease in pregnancy: a systematic overview from 1978 through 1996. J Am Coll Cardiol 1998; 31: 1650-1657.

4 Galiè N, Hoeper MM, Humbert M, et al. Guidelines for the diagnosis and treatment of pulmonary hypertension. Eur Respir $J$ 2009; 34: 1219-1263.

5 McLaughlin VV, Archer SL, Badesch DB, et al. ACCF/AHA 2009 expert consensus document on pulmonary hypertension: a report of the American College of Cardiology Foundation Task Force on expert consensus documents and the American Heart Association: Developed in collaboration with the American College of Chest Physicians, American Thoracic Society, Inc., and the Pulmonary Hypertension Association. Circulation 2009; 119: 2250-2294.

6 Gomberg-Maitland M, Olschewski H. Prostacyclin therapies for the treatment of pulmonary arterial hypertension. Eur Respir $J$ 2008; 31: 891-901.

7 Wilkins MR, Wharton J, Grimminger F, et al. Phosphodiesterase inhibitors for the treatment of pulmonary hypertension. Eur Respir J 2008; 32: 198-209.

8 Dupuis J, Hoeper MM. Endothelin receptor antagonists in pulmonary arterial hypertension. Eur Respir J 2008; 31: 407-415.

9 Humbert M, Sitbon O, Yaïci A, et al. Survival in incident and prevalent cohorts of patients with pulmonary arterial hypertension. Eur Respir J 2010; 36: 549-555.

10 Galie N, Manes A, Negro L, et al. A meta-analysis of randomized controlled trials in pulmonary arterial hypertension. Eur Heart J 2009; 30: 394-403.

11 Sitbon $\mathrm{O}$, Humbert $\mathrm{M}$, Jais $\mathrm{X}$, et al. Long-term response to calcium channel blockers in idiopathic pulmonary arterial hypertension. Circulation 2005; 111: 3105-3111.

12 Bonnin M, Mercier FJ, Sitbon O, et al. Severe pulmonary hypertension during pregnancy: mode of delivery and anesthetic management of 15 consecutive cases. Anesthesiology 2005; 102: 1133-1137.

13 Bédard E, Dimopoulos K, Gatzoulis MA. Has there been any progress made on pregnancy outcomes among women with pulmonary arterial hypertension? Eur Heart J 2009; 30: 256-265.

14 Kiely DG, Condliffe R, Webster V, et al. Improved survival in pregnancy and pulmonary hypertension using a multiprofessional approach. Brit J Obstet Gynaec 2010; 117: 565-574.

15 Barst RJ, Rubin LJ, Long WA, et al. A comparison of continuous intravenous epoprostenol (prostacyclin) with conventional therapy for primary pulmonary hypertension. The Primary Pulmonary Hypertension Study Group. N Engl J Med 1996; 334: 296-302.

16 Galie N, Ghofrani HA, Torbicki A, et al. Sildenafil citrate therapy for pulmonary arterial hypertension. N Engl J Med 2005; 353: 2148-2157.

17 Rubin LJ, Badesch DB, Barst RJ, et al. Bosentan therapy for pulmonary arterial hypertension. N Engl J Med 2002; 346: 896-903.

18 Roberts NV, Keast PJ. Pulmonary hypertension and pregnancy: a lethal combination. Anaesth Intensive Care 1990; 18: 366-374.

19 Hickey PR, Retzack SM. Acute right ventricular failure after pulmonary hypertensive responses to airway instrumentation: effect of fentanyl dose. Anesthesiology 1993; 78: 372-376.

20 Kerbaul F, Rondelet B, Motte S, et al. Isoflurane and desflurane impair right ventricular-pulmonary arterial coupling in dogs. Anesthesiology 2004; 101: 1357-1362.

21 Blaise G, Langleben D, Hubert B. Pulmonary arterial hypertension: pathophysiology and anesthetic approach. Anesthesiology 2003; 99: 1415-1432.

22 Moudgil R, Michelakis ED, Archer SL. Hypoxic pulmonary vasoconstriction. J Appl Physiol 2005; 98: 390-403.

23 Chakravarty EF, Khanna D, Chung L. Pregnancy outcomes in systemic sclerosis, primary pulmonary hypertension, and sickle cell disease. Obstet Gynecol 2008; 111: 927-934.

24 Treinen KA, Louden C, Dennis MJ, et al. Developmental toxicity and toxicokinetics of two endothelin receptor antagonists in rats and rabbits. Teratology 1999; 59: 51-59. 